\title{
Gamma-ray Observation of the Cygnus Region with the Tibet Air Shower Array
}

Y, Katayose ${ }^{a, *}$ and T. K. Sako ${ }^{b}$ on behalf of the Tibet AS $\gamma$ Collaboration

(a complete list of authors can be found at the end of the proceedings)

${ }^{a}$ Faculty of Engineering, Yokohama National University, 79-5 Tokiwadai, Hodogaya-ku, Yokohama, Japan

${ }^{b}$ Institute for Cosmic Ray Research, University of Tokyo, 5-1-5 Kashiwanoha, Kashiwa, Japan

E-mail: katayose-yusaku-dv@ynu.ac..jp, tsako@icrr.u-tokyo.ac.jp

The Tibet-III air shower array and underground water-Cherenkov-type muon detector array have been successfully operated since 2014 , at an altitude of 4,300m in Tibet, China. The gamma-ray energy and arrival direction are determined by the Tibet AS array, while the MD array enables us to suppress more than $99.9 \%$ of background cosmic rays above $100 \mathrm{TeV}$, using counting number of muons in an air shower at 2.4 m underground.

We surveyed gamma-ray emissions in the energy region above $10 \mathrm{TeV}$ from the Cygnus region in our Galaxy. Two sources were significantly detected in the directions of the Cygnus OB1 and OB2 associations. One of the gamma-ray emissions had an excess of pre-trial(post-trial) detection significance of $5.3 \sigma(4.7 \sigma)$ in the direction centered on (R.A., Dec) $=\left(308.04^{\circ} \pm\right.$ $0.08^{\circ}, 41.46^{\circ} \pm 0.06^{\circ}$ ), which was associated with Pulsar PSR J2032 + 4127. The obtained spectrum from $10 \mathrm{TeV}$ to120 $\mathrm{TeV}$ can be expressed by a simple power-law as $d F / d E=(4.13 \pm 0.83) \times$ $10^{-16}(E / 40 \mathrm{TeV})^{3.12 \pm 0.12} \mathrm{TeV}^{-1} \mathrm{~cm}^{-2} \mathrm{~s}^{-1}$. The other had an excess of pre-trial(post-trial) detection significance of $6.7 \sigma(6.2 \sigma)$ in the direction centered on (R.A., Dec) $=\left(304.99^{\circ} \pm 0.11^{\circ}, 36.84^{\circ} \pm\right.$ $0.08^{\circ}$ ), which was associated mainly with a pulsar wind nebula PWN G75.2+0.1 with the pulsar moving away from its original birthplace situated around the centroid of the observed gamma-ray emission. The obtained spectrum from $10 \mathrm{TeV}$ to $200 \mathrm{TeV}$ can be expressed by a simple power-law as $d F / d E=(10.6 \pm 1.3) \times 10^{-16}(E / 40 \mathrm{TeV})^{2.70 \pm 0.13} \mathrm{TeV}^{-1} \mathrm{~cm}^{-2} \mathrm{~s}^{-1}$, or including an exponential cutoff as $d F / d E=(3.6 \pm 2.0) \times 10^{-15}(E / 40 \mathrm{TeV})^{1.60 \pm 0.5} \exp (E /(44 \pm 21 \mathrm{TeV})) \mathrm{TeV}^{-1} \mathrm{~cm}^{-2} \mathrm{~s}^{-1}$.

$37^{\text {th }}$ International Cosmic Ray Conference (ICRC 2021)

July 12th - 23rd, 2021

Online - Berlin, Germany

\footnotetext{
${ }^{*}$ Presenter
} 


\section{Introduction}

The Cygnus region located in the direction of the Cygnus constellation is a huge star-forming region, and there are many energetic objects such as the Pulsar Nebula, SNR, Wolf-Rayet stars, open cluster, and $\mathrm{OB}$ associations, etc. This region is a natural laboratory to study cosmicray acceleration and transport and has been observed in various wavebands. In particular, the Cygnus-OB1 and Cygnus-OB2 regions, which have many massive and hot stars, are the sources of $\mathrm{TeV}$ gamma rays. The first reported $\mathrm{TeV}$ gamma-ray source from Cygnus OB2 was J2032+ 4130 [1, 2], discovered by the HEGRA Imaging Air Cherenkov Telescope (IACT) and confirmed by Whipple [3], MAGIC [4], and VERITAS [5, 6]. According to their report, the gamma-ray intensity is roughly $3 \%$ of Crab nebula, and the spread of the source is about $0.1^{\circ}$ to $0.2^{\circ}$. On the other hand, surface air shower array experiments report higher gamma-ray intensities than the IACTs' results in the multi-TeV energy region [7-11]. The flux discrepancy measured by IACT and the air shower array may be due to the morphological complexity of this region caused by the presence of gamma-ray sources such as Cygnus cocoon etc. The Fermi satellite-mounted LAT reported the existence of a region with strong gamma-ray intensity called "Cygnus cocoon" nearby Cygnus OB2. This is a star-forming region, and cosmic rays generated by star-forming activity are thought to be confined and accelerated in the interstellar space [12]. Cygnus Cocoon has a shape that connects Cygnus OB2 and NGC 6910, which is an open cluster and has a size of about $50 \mathrm{pc}$. It overlaps with the region of Cygnus OB2, which complicates morphology. The gamma-ray sources in this region are considered to be PWNs of PSR J2032+4127 and PSR J2021+4026 or SNR in Cygnus Cocoon, but the details are under debate.

In the Cygnus OB1 direction, the Milagro experiment discovered the MGRO J2019+37, which emits gamma rays exceeding $10 \mathrm{TeV}$ [13], and was confirmed in the HAWC [14, 15] experiment. Also, VERITAS observed the same region above $0.6 \mathrm{TeV}$ and separated the gamma-ray emissions into two sources: VER J2019+368 and VER J2016+371 [16]. At $0.36^{\circ}$ east of the center of gravity of VER J2019 +368, there is a radio pulsar PSR J2021+3651 [17]. PSR J2021+3651 is one of the relatively few pulsars with gamma-ray pulsations observed by Fermi-LAT [18], and also appears to form the pulsar wind nebula referred to as PWN G75.2+0.1 [19]. The radio and X-ray morphologies of this nebula feature a bright bow-shaped tail extending westwards from the pulsar, indicating that the pulsar is moving eastwards with its birthplace as far west as the apparent end of the tail at $0.2^{\circ}$ west of the current pulsar position [16, 20]. PSR J2021+3651/PWN G75.2+0.1 is considered to be the most promising object for VER J2019+368.

The Tibet air shower array has a high sensitivity for gamma rays in the region of $10 \mathrm{TeV}$ or more and has a wide field of view of about 2 rad. To examine the morphology and the spectrum of gamma-ray in the higher-energy region, we searched for gamma-ray emissions from the Cygnus region containing $\mathrm{OB} 1$ and $\mathrm{OB} 2$ associations with the data measured by the TibetAS $\gamma$ experiment.

\section{Tibet AS $\gamma$ experiment}

The Tibet air-shower (AS) array has been observing cosmic rays and gamma rays above $\mathrm{TeV}$ energies since 1990 at Yangbajing $\left(90.522^{\circ} \mathrm{E}, 30.102^{\circ} \mathrm{N} ; 4300 \mathrm{~m}\right.$ above sea level) in Tibet, China [21]. In this work, we use data obtained by the Tibet air shower array combined with the 
muon detector array during 719 live days from 2014 February to 2017 May. Our data analysis method and data selection criteria as well as the array configuration are the same as described in our previous papers [22, 23].

\section{Data analysis and Results}

We used the Equi-Zenith-Angle method [24] to estimate the gamma-ray excess count and the number of background events. Twenty OFF regions were taken, and the radius of the analysis window was variable depending on the recorded air-shower size from approximately $0.7^{\circ}$ at lower energies around $10 \mathrm{TeV}$ to a lower limit of $0.5^{\circ}$. The intensity distribution of gamma-ray in an area of (R.A., Dec.) from $\left(295^{\circ}, 30^{\circ}\right)$ to $\left(315^{\circ}, 45^{\circ}\right)$ was examined and two gamma-ray sources were detected significantly above $10 \mathrm{TeV}$ in the directions of Cygnus OB1 and OB2 respectively.

\subsection{Cygnus OB2}

Figure 1(a) shows a detection significance map around the gamma-ray source detected by this work with photon energies above $10 \mathrm{TeV}$ in the direction of Cygnus OB2. The sky is gridded in $0.1^{\circ} \times 0.1^{\circ}$ pixels and the significance value of each pixel calculated according to [25] is smoothed by a circular search window of radius, $R_{w}$ centered at the pixel. Assuming a symmetrical $2 \mathrm{D}$ Gaussian distribution for the gamma-ray excess, we fit the events within the $4^{\circ} \times 4^{\circ}$ region around the source using the unbinned maximum likelihood method. The centroid of gamma-ray emissions detected at the pre-trial (post-trial) detection significance of $5.3 \sigma(4.7 \sigma)$ above $10 \mathrm{TeV}$ is estimated at $($ R.A., Dec. $)=\left(308.04^{\circ} \pm 0.08^{\circ}, 41.46^{\circ} \pm 0.06^{\circ}\right)$. We name this source TASG J2032+414. The location of TASG J2032+414 is in good agreement with that of the pulsar PSR J2032+4127 and consistent with that of HAWC J2031+415 [26] at the $1.7 \sigma$ level, while it appears to deviate from that of $\mathrm{TeV} \mathrm{J} 2032+4130$ reported in [27] at the $2.8 \sigma$ level.

Figure 1(b) shows the distribution of the number of events observed with photon energies above $10 \mathrm{TeV}$ as a function of the square of the opening angle $\phi$ between the estimated arrival direction and the centroid of TASG J2032+414. To estimate a possible source extension, we perform the $\chi^{2}$ fitting of the data with the function $A \exp \left[-\phi^{2} / 2\left(\sigma_{\mathrm{PSF}}^{2}+\sigma_{\mathrm{EXT}}^{2}\right)\right]+N_{\mathrm{BG}}$ where $A$ and $\sigma_{\mathrm{EXT}}$ are two fitting parameters and $\sigma_{\mathrm{PSF}}=0.36^{\circ}$ and $N_{\mathrm{BG}}=224.5$ are the point spread function (PSF) of our instrument above $10 \mathrm{TeV}$ and the number of background events estimated from the background cosmic-ray data, we get $\sigma_{\mathrm{EXT}}=0.00^{\circ} \pm 0.14^{\circ}$, which is consistent with that obtained from the maximum likelihood fitting described above. The $\chi^{2} /$ ndf of the fitting is $33.8 / 38$. With a large error of $0.14^{\circ}$, the $\sigma_{\mathrm{EXT}}$ value above $10 \mathrm{TeV}$ does not indicate whether TASG J2032+414 is extended or not even though it is consistent with the previous measurements at multi-TeV energies by IACTs, ARGO and HAWC within the $2 \sigma$ level $[2,4,6,9,26,27]$. Figure 2 shows the differential energy spectrum of TASG J2032+414. Although there is a discrepancy in flux at multi-TeV energies as explained in the Introduction, our flux data points above $10 \mathrm{TeV}$ are consistent with previous measurements of IACTs when the spill-over of gamma-ray signals outside their integration radius is taken into account. Our spectrum from $10 \mathrm{TeV}$ to $120 \mathrm{TeV}$ can be expressed by a simple power-law as $d F / d E=N_{0}(E / 40 \mathrm{TeV})^{-\Gamma}$ where $N_{0}=(4.13 \pm 0.83) \times 10^{-16} \mathrm{TeV}^{-1} \mathrm{~cm}^{-2} \mathrm{~s}^{-1}$ is the differential gamma-ray flux at $40 \mathrm{TeV}$ and $\Gamma=3.12 \pm 0.21$ is the spectral index $\left(\chi^{2} / \mathrm{ndf}=1.6 / 4\right)$. Unfortunately, the time span of our data does not cover the 2017 autumn periastron period of the binary system 
PSR J2032+4127/MT91 213 when a significant flare was detected in the TeV gamma-ray flux [27]. We find no significant flux variability in the time span of our data.
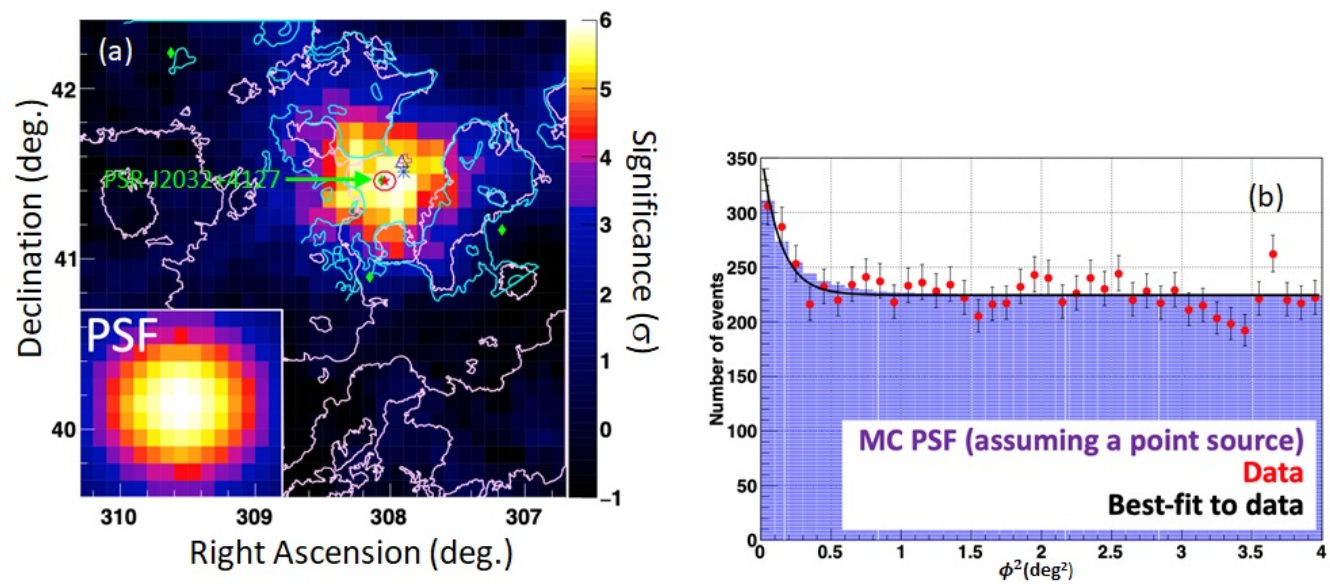

Figure 1: The gamma-ray emission sources detected above $10 \mathrm{TeV}$ in the directions of Cygnus OB2. The left figure is a significance map around the source, smoothed by search windows. The point spread function (PSF) is shown in the inset figure. The red-filled star with a position error circle is the centroid of TASG J2032+414 obtained by this work, while the magenta open cross is the centroid of VER J2031+415, and the blue asterisk is that of HAWC J2031+415. The green-filled diamonds show Fermi-LAT sources. The blue open triangle indicates the centroid of MAGIC J2031+4134. The green-filled diamond coincident with our gamma-ray emission centroid is the pulsar PSR J2032+4127. The sky-blue contours indicate $1420 \mathrm{MHz}$ radio emissions provided by the Canadian Galactic Plane Survey, and the pink contours indicate $24 \mu \mathrm{m}$ infrared emissions by the Cygnus-X Spitzer Legacy Survey [28, 29]. The right figure shows the distribution of the number of events observed with photon energies above $10 \mathrm{TeV}$ as a function of the square of the opening angle between the estimated arrival direction and the centroid of TASG J2032+414. The red-filled circles are the experimental data, with the best fit Gaussian function indicated by the solid line. The blue histogram is the distribution of events expected by the MC simulation assuming a point-like gamma-ray source.

\subsection{Cygnus OB1}

Figure 3(a) shows a significance map above $10 \mathrm{TeV}$ in the direction of Cygnus OB1 obtained by this work. The centroid of gamma-ray emissions is estimated at (R.A., Dec.) $=\left(304.99^{\circ} \pm\right.$ $0.11^{\circ} 36.84^{\circ} \pm 0.08^{\circ}$ ) with the pre-trial (post-trial) detection significance of $6.7 \sigma(6.2 \sigma)$. We name this source TASG J2019+368. The centroid of TASG J2019+368 is consistent with that reported by HAWC [15] within the $1 \sigma$ level, and by VERITAS [6] within the $2 \sigma$ level. The pulsar PSR J2021+3651, located $0.23^{\circ}$ east of the TASG J2019+368 centroid, has a nebula extending westwards from the pulsar, PWN G75.2+0.1, which is coincident with the location of TASG J2019+368. Figure 3(b) shows the angular distribution of the events observed above $10 \mathrm{TeV}$. The experimental data can be fitted with a Gaussian function with a source extension of $\sigma_{\mathrm{EXT}}=0.28^{\circ} \pm 0.07^{\circ}$ above 10 $\mathrm{TeV}$, consistent with the extension reported by VERITAS [6] (HAWC [14]) at the $2.1 \sigma(0.3 \sigma)$ level. The $\chi^{2} /$ ndf of the fitting is $49.1 / 38$. Figure 4 shows the differential gamma-ray energy spectrum of TASG J2019+368, which is in good agreement with the HAWC spectrum and consistently connects 


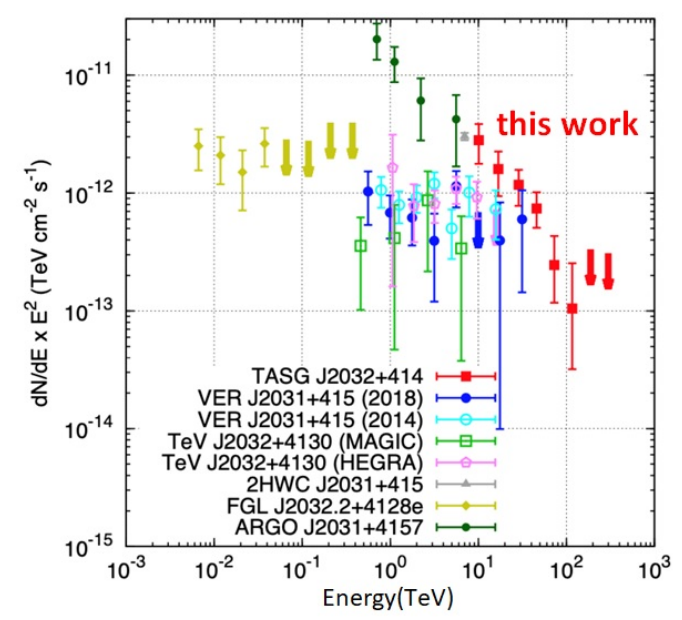

Figure 2: Differential gamma-ray energy spectra of TASG J2032+414 with 95\% C.L. upper limits measured by this work (red filled squares/arrows). The blue-filled circles/arrows (sky-blue open circles) show the gamma-ray spectrum reported by VERITAS in 2018 (2014) [5, 6], the gray filled triangle by HAWC [11], and the dark-green filled circles by ARGO [9]. Additionally, the gold-filled diamonds are reported by Fermi-LAT cite6, the green open squares by MAGIC [4], and the pink pentagons/arrow by HEGRA [2].

with the VERITAS spectrum reported in 2014. Our spectrum can be expressed either as $d F / d E=$ $N_{0}(E / 40 \mathrm{TeV})^{-\Gamma}$ with $N_{0}=(10.6 \pm 1.3) \times 10^{16} \mathrm{TeV}^{-1} \mathrm{~cm}^{-2} \mathrm{~s}^{-1}$ and $\Gamma=2.70 \pm 0.13\left(\chi^{2} / \mathrm{ndf}=10.4 / 5\right)$ , or including an exponential cutoff as $d F / d E=N_{0}(E / 40 \mathrm{TeV})^{-\Gamma} \exp \left(E / E_{\text {cut }}\right)$ with $N_{0}=(3.6 \pm$ 2.0) $\times 10^{-15} \mathrm{TeV}^{-1} \mathrm{~cm}^{-2} \mathrm{~s}^{-1}, \Gamma=1.6 \pm 0.5$ and $E_{\text {cut }}=44 \pm 21 \mathrm{TeV}\left(\chi^{2} / \mathrm{ndf}=3.0 / 4\right)$.

\section{Conclusion}

Using the Tibet air shower array combined with the underground muon detector array, we have detected two gamma-ray sources in the direction of the Cygnus region significantly with photon energies above $10 \mathrm{TeV}$. The location of TASG J2032+414 is coincident with PSR J2032+4127, and that of TASG J2019+368 is coincident with PWN G75.2+0.1. We provide for the first time flux data points from $40 \mathrm{TeV}$ to $120 \mathrm{TeV}$ for the gamma-ray emission region in the direction of Cygnus $\mathrm{OB} 2$, and we confirm the HAWC spectrum up to $100 \mathrm{TeV}$ and provide flux data points from 120 $\mathrm{TeV}$ to $200 \mathrm{TeV}$ for the gamma-ray emission region in the direction of Cygnus OB1. For both cases, the observed gamma-ray emissions likely result from relativistic electrons injected by the pulsar through the ICS with synchrotron and ambient photons.

\section{Acknowledgements}

The collaborative experiment of the Tibet Air Shower Arrays has been conducted under the auspices of the Ministry of Science and Technology of China and the Ministry of Foreign Affairs of Japan. This work was supported in part by a Grant-in-Aid for Scientific Research on Priority Areas from the Ministry of Education, Culture, Sports, Science and Technology, and by Grants-in-Aid for Science Research from the Japan Society for the Promotion of Science in Japan. This work is supported by the National Key R\&D Program of China (No. 2016YFE0125500), the Grants from the 

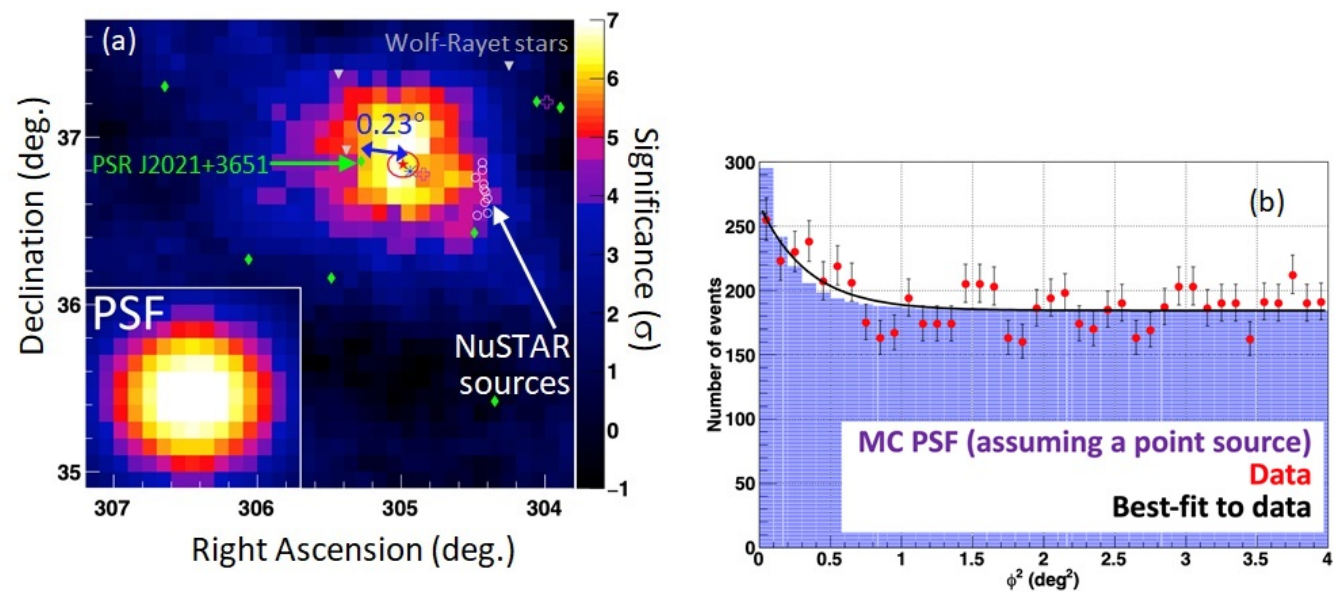

Figure 3: The gamma-ray emission sources detected above $10 \mathrm{TeV}$ in the directions of Cygnus OB1. The left figure is a significance map around the source. The red-filled star with a position error circle is the centroid of TASG J2019+368 obtained by this work. The white open circles are NuSTAR X-ray sources [30], and the gray-filled inverted triangles are Wolf-Rayet stars [31]. The green-filled diamond located at $0.23^{\circ}$ east of our emission centroid is the pulsar PSR J2021+3651. The magenta open cross located at (R.A., Dec.) $=\left(303.99^{\circ}, 37.21^{\circ}\right)$ is another VERITAS source VER J2016+371 [6]s, which is not detected significantly in this work.The right figure shows the distribution of the number of events observed with photon energies above $10 \mathrm{TeV}$ as a function of the square of the opening angle between the estimated arrival direction and the centroid of TASG J2019+368.

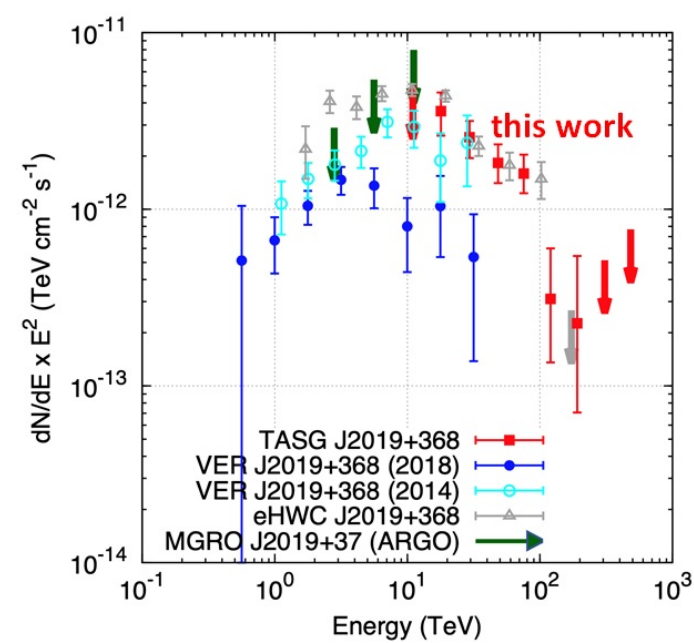

Figure 4: Differential gamma-ray energy spectra of TASG J2019+368 with 95\% C.L. upper limits measured by this work (red-filled squares/arrows). The blue-filled circles(sky-blue open circles) show the gamma-ray spectrum reported by VERITAS in 2018 (2014) [6, 16], the gray open triangles/arrow by HAWC [11, 14], and dark-green filled arrow by ARGO [9].

National Natural Science Foundation of China (Nos. 11533007, 11673041, 11873065, 11773019, 11773014, 11633007, 11803011, and 11851305), and the Key Laboratory of Particle Astrophysics, 
Institute of High Energy Physics, CAS. This work is also supported by the joint research program of the Institute for Cosmic Ray Research (ICRR), the University of Tokyo.

\section{References}

[1] F. Aharonian et al., Astron. Astrophys. 393, L37 (2002).

[2] F. Aharonian et al., Astron. Astrophys. 431, 197 (2005).

[3] A. Konopelko et al., Astrophys. J. 658, 1062 (2007).

[4] J. Albert et al., Astrophys. J. Lett. 675, L25 (2008).

[5] E. Aliu et al., Astrophys. J. 783, 16 (2014).

[6] A. U. Abeysekara et al., Astrophys. J. 861, 134 (2018).

[7] A. A. Abdo et al., Astrophys. J. Lett. 664, L91 (2007).

[8] A. A. Abdo et al., Astrophys. J. 753, 159 (2012).

[9] B. Bartoli et al., Astrophys. J. Lett. 745, L22 (2012).

[10] B. Bartoli et al., Astrophys. J. 790, 152 (2014).

[11] A. U. Abeysekara et al., Astrophys. J. 843, 40 (2017).

[12] M. Ackermann et al., Science 334, 1103 (2011).

[13] A. A. Abdo et al., Astrophys. J. Lett. 658, L33 (2007).

[14] A. U. Abeysekara et al., Phys. Rev. Lett. 124, 021102 (2020).

[15] A. Albert et al., Astrophys. J. 905, 76 (2020).

[16] E. Aliu et al., Astrophys. J. 788, 78 (2014).

[17] M. S. E. Roberts, J. W. T. Hessels, S. M. Ransom, V. M. Kaspi, P. C. C. Freire, F. Crawford, and D. R. Lorimer, Astrophys. J. Lett. 577, L19 (2002).

[18] A. A. Abdo et al., Astrophys. J. 700, 1059 (2009).

[19] J. W. T. Hessels, M. S. E. Roberts, S. M. Ransom, V. M. Kaspi, R. W. Romani, C.-Y. Ng, P. C. C. Freire, and B. M. Gaensler, Astrophys. J. 612, 389 (2004).

[20] T. Mizuno, N. Tanaka, H. Takahashi, J. Katsuta, K.Hayashi, and R. Yamazaki, Astrophys. J. 841, 104 (2017).

[21] M. Amenomori et al., Phys. Rev. Lett. 69, 2468 (1992).

[22] M. Amenomori et al., Phys. Rev. Lett. 123, 051101 (2019). 
[23] M. Amenomori et al., Nat. Astron. Let. (2021) https://doi.org/10.1038/s41550-020-01294-9.

[24] M. Amenomori et al., Astrophys. J. 633, 1005 (2005).

[25] T.-P. Li and Y.-Q. Ma, Astrophys. J. 272, 317 (1983).

[26] A. U. Abeysekara et al., Nat. Astron. Let. (2021) https://doi.org/10.1038/s41550-021-01318-y.

[27] A. U. Abeysekara et al., Astrophys. J. Lett. 867, L19 (2018).

[28] I. M. Beerer et al., Astrophys. J. 720, 679 (2010).

[29] K. E. Kraemer et al., Astron. J. 139, 2319 (2010).

[30] E. V. Gotthelf et al., Astrophys. J. 826, 25 (2016).

[31] K. A. van der Hucht, New Astron. Rev. 45, 135 (2001).

\section{Full Authors List: Tibet AS $\gamma$ Collaboration}

M. Amenomori ${ }^{1}$, S. Asano ${ }^{2}$, Y. W. Bao ${ }^{3}$, X. J. Bi ${ }^{4}$, D. Chen ${ }^{5}$, T. L. Chen ${ }^{6}$, W. Y. Chen ${ }^{4}, \mathrm{Xu} \mathrm{Chen}^{4}, \mathrm{Y}_{\mathrm{C}} \mathrm{Chen}^{3}, \mathrm{Cirennima}^{6}$, S. W. Cui ${ }^{7}$, Danzengluobu $^{6}$, L. K. Ding ${ }^{4}$, J. H. Fang ${ }^{4,8}$, K. Fang ${ }^{4}$, C. F. Feng ${ }^{9}$, Zhaoyang Feng ${ }^{4}$, Z. Y. Feng ${ }^{10}$, Qi Gao ${ }^{6}$, A. Gomi ${ }^{11}$, Q. B. Gou ${ }^{4}$,

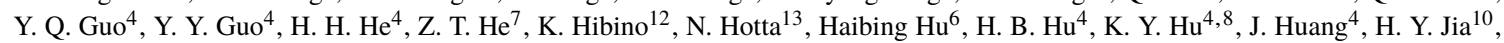
L. Jiang ${ }^{4}$, P. Jiang 5 , H. B. Jin ${ }^{5}$, K. Kasahara ${ }^{14}$, Y. Katayose ${ }^{11}$, C. Kato ${ }^{2}$, S. Kato ${ }^{15}$, T. Kawashima ${ }^{15}$, K. Kawata ${ }^{15}$, M. Kozai ${ }^{16}$, D. Kurashige ${ }^{11}$, Labaciren ${ }^{6}$, G. M. Le ${ }^{17}$, A. F. Li ${ }^{18,9,4}$, H. J. Li ${ }^{6}$, W. J. Li ${ }^{4,10}$, Y. Li ${ }^{5}$, Y. H. Lin ${ }^{4,8}$, B. Liu ${ }^{19}$, C. Liu ${ }^{4}$, J. S. Liu ${ }^{4}$,

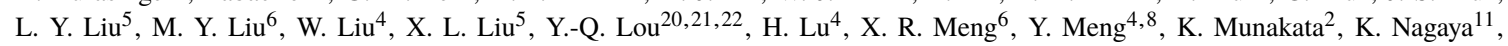
Y. Nakamura ${ }^{15}$, Y. Nakazawa ${ }^{23}$, H. Nanjo ${ }^{1}$, C. C. Ning $^{6}$, M. Nishizawa ${ }^{24}$, M. Ohnishi ${ }^{15}$, S. Okukawa ${ }^{11}$, S. Ozawa ${ }^{25}$, L. Qian $^{5}$, X. Qian ${ }^{5}$, X. L. Qian $^{26}$, X. B. Qu $^{27}$, T. Saito ${ }^{28}$, Y. Sakakibara ${ }^{11}$, M. Sakata ${ }^{29}$, T. Sako ${ }^{15}$, T. K. Sako ${ }^{15}$, J. Shao ${ }^{4,9}$, M. Shibata ${ }^{11}$, A. Shiomi ${ }^{23}$, H. Sugimoto ${ }^{30}$, W. Takano ${ }^{12}$, M. Takita ${ }^{15}$, Y. H. Tan ${ }^{4}$, N. Tateyama ${ }^{12}$, S. Torii ${ }^{31}$, H. Tsuchiya $^{32}$, S. Udo ${ }^{12}$, H. Wang $^{4}$, Y. P. Wang ${ }^{6}$, Wangdui ${ }^{6}$, H. R. $\mathrm{Wu}^{4}$, Q. $\mathrm{Wu}^{6}$, J. L. Xu ${ }^{5}$, L. Xue ${ }^{9}$, Z. Yang ${ }^{4}$, Y. Q. Yao ${ }^{5}$, J. Yin ${ }^{5}$, Y. Yokoe ${ }^{15}$, N. P. Yu ${ }^{5}$, A. F. Yuan ${ }^{6}$, L. M. Zhai ${ }^{5}$, C. P. Zhang ${ }^{5}$, H. M. Zhang ${ }^{4}$, J. L. Zhang ${ }^{4}$, X. Zhang ${ }^{3}$, X. Y. Zhang ${ }^{9}$, Y. Zhang ${ }^{4}$, Yi Zhang ${ }^{33}$, Ying Zhang ${ }^{4}$, S. P. Zhao ${ }^{4}$, Zhaxisangzhu $^{6}$ and X. X. Zhou ${ }^{10}$

${ }^{1}$ Department of Physics, Hirosaki University, Hirosaki 036-8561, Japan. ${ }^{2}$ Department of Physics, Shinshu University, Matsumoto 390-8621, Japan. ${ }^{3}$ School of Astronomy and Space Science, Nanjing University, Nanjing 210093, China. ${ }^{4}$ Key Laboratory of Particle Astrophysics, Institute of High Energy Physics, Chinese Academy of Sciences, Beijing 100049, China. ${ }^{5}$ National Astronomical Observatories, Chinese Academy of Sciences, Beijing 100012, China. ${ }^{6}$ Department of Mathematics and Physics, Tibet University, Lhasa 850000, China. ${ }^{7}$ Department of Physics, Hebei Normal University, Shijiazhuang 050016, China. ${ }^{8}$ University of Chinese Academy of Sciences, Beijing 100049, China. ${ }^{9}$ Institute of Frontier and Interdisciplinary Science and Key Laboratory of Particle Physics and Particle Irradiation (MOE), Shandong University, Qingdao 266237, China. ${ }^{10}$ Institute of Modern Physics, SouthWest Jiaotong University, Chengdu 610031, China. ${ }^{11}$ Faculty of Engineering, Yokohama National University, Yokohama 240-8501, Japan. ${ }^{12}$ Faculty of Engineering, Kanagawa University, Yokohama 221-8686, Japan. ${ }^{13}$ Faculty of Education, Utsunomiya University, Utsunomiya 3218505, Japan. ${ }^{14}$ Faculty of Systems Engineering, Shibaura Institute of Technology, Omiya 330-8570, Japan. ${ }^{15}$ Institute for Cosmic Ray Research, University of Tokyo, Kashiwa 277-8582, Japan. ${ }^{16}$ Institute of Space and Astronautical Science, Japan Aerospace Exploration Agency (ISAS/JAXA), Sagamihara 252-5210, Japan. ${ }^{17}$ National Center for Space Weather, China Meteorological Administration, Beijing 100081, China. ${ }^{18}$ School of Information Science and Engineering, Shandong Agriculture University, Taian 271018, China. ${ }^{19}$ Department of Astronomy, School of Physical Sciences, University of Science and Technology of China, Hefei 230026, China. ${ }^{20}$ Department of Physics and Tsinghua Centre for Astrophysics (THCA), Tsinghua University, Beijing 100084, China. ${ }^{21}$ Tsinghua University-National Astronomical Observatories of China (NAOC) Joint Research Center for Astrophysics, Tsinghua University, Beijing 100084, China. ${ }^{22}$ Department of Astronomy, Tsinghua University, Beijing 100084, China. ${ }^{23}$ College of Industrial Technology, Nihon University, Narashino 275-8576, Japan. ${ }^{24}$ National Institute of Informatics, Tokyo 101-8430, Japan. ${ }^{25}$ National Institute of Information and Communications Technology, Tokyo 184-8795, Japan. ${ }^{26}$ Department of Mechanical and Electrical Engineering, Shangdong Management University, Jinan 250357, China. ${ }^{27}$ College of Science, China University of Petroleum, Qingdao 266555, China. ${ }^{28}$ Tokyo Metropolitan College of Industrial Technology, Tokyo 116-8523, Japan. ${ }^{29}$ Department of Physics, Konan University, Kobe 658-8501, Japan. ${ }^{30}$ Shonan Institute of Technology, Fujisawa 251-8511, Japan. ${ }^{31}$ Research Institute for Science and Engineering, Waseda University, Tokyo 162-0044, Japan. ${ }^{32}$ Japan Atomic Energy Agency, Tokai-mura 319-1195, Japan. ${ }^{33}$ Key Laboratory of Dark Matter and Space Astronomy, Purple Mountain Observatory, Chinese Academy of Sciences, Nanjing 210034, China. 